\title{
Understanding the Relationship Between Co-occurring PTSD and MDD: Symptom Severity and Affect
}

\author{
Loren M. Post ${ }^{\mathrm{a}}$, Lori A. Zoellner ${ }^{\mathrm{b}}$, Eric Youngstrom ${ }^{\mathrm{c}}$, and Norah C. Feeny ${ }^{\mathrm{a}}$ \\ aCase Western Reserve University, Department of Psychological Sciences, 10900 Euclid Ave., \\ Cleveland, $\mathrm{OH}$ 44106, USA \\ bUniversity of Washington, Department of Psychology, Box 351525, Seattle, WA 98195, USA \\ 'University of North Carolina at Chapel Hill, Department of Psychology, CB\#3270 Davie Hall, \\ Chapel Hill, NC 27599, USA
}

\begin{abstract}
How to best understand theoretically the nature of the relationship between co-occurring PTSD and MDD (PTSD+MDD) is unclear. In a sample of 173 individuals with chronic PTSD, we examined whether the data were more consistent with current co-occurring MDD as a separate construct or as a marker of posttraumatic stress severity, and whether the relationship between PTSD and MDD is a function of shared symptom clusters and affect components. Results showed that the more severe depressive symptoms found in PTSD+MDD as compared to PTSD remained after controlling for PTSD symptom severity. Additionally, depressive symptom severity significantly predicted co-occurring MDD even when controlling for PTSD severity. In comparison to PTSD, PTSD+MDD had elevated dysphoria and re-experiencing - but not avoidance and hyperarousal - PTSD symptom cluster scores, higher levels of negative affect, and lower levels of positive affect. These findings provide support for PTSD and MDD as two distinct constructs with overlapping distress components.
\end{abstract}

\section{Keywords}

posttraumatic stress disorder; major depressive disorder; co-occurrence; comorbidity; positive affect; negative affect

\begin{abstract}
A large body of evidence suggests that posttraumatic stress disorder (PTSD) and major depressive disorder (MDD) commonly co-occur (Breslau, Davis, Andreski, \& Peterson, 1991; Brown, Campbell, Lehman, Grisham, \& Mancill, 2001; Davidson, Hughes, Blazer, \& George, 1991; Helzer, Robins, \& McEvoy, 1987; Kessler, Berglund, Demler, Jin, \& Walters, 2005; Kessler, Sonnega, Bromet, Hughes, \& Nelson, 1995). Indeed, the National Comorbidity Survey-Revised (NCS-R) showed a .50 correlation between lifetime PTSD and lifetime MDD (Kessler, Berglund et al., 2005). Large-scale studies of civilian and veteran samples show that as many as $51 \%$ to $82 \%$ of individuals with current PTSD also endorse
\end{abstract}

(C) 2011 Elsevier Ltd. All rights reserved.

Correspondence concerning this article should be addressed to Loren M. Post, Case Western Reserve University, Department of Psychological Sciences, 10900 Euclid Ave., Cleveland, OH 44106, USA, loren.post@ case.edu Phone: 216-368-8934 EXT 4 Fax: 216-368-4891.

Publisher's Disclaimer: This is a PDF file of an unedited manuscript that has been accepted for publication. As a service to our customers we are providing this early version of the manuscript. The manuscript will undergo copyediting, typesetting, and review of the resulting proof before it is published in its final citable form. Please note that during the production process errors may be discovered which could affect the content, and all legal disclaimers that apply to the journal pertain. 
current co-occurring MDD (Hankin, Spiro, Miller, \& Kazis, 1999; Shore, Vollmer, \& Tatum, 1989). PTSD has also been shown to increase the risk for first onset of MDD (Breslau, Davis, Peterson, \& Schultz, 1997; Kessler et al., 1995).

The co-occurrence of PTSD and MDD is associated with greater disorder severity (e.g., Kessler, Chiu, Demler, \& Walters, 2005). Individuals with co-occurring PTSD and MDD tend to display more anxiety, depression, and PTSD symptoms, lower global functioning (Blanchard, Buckley, Hickling, \& Taylor, 1998; Momartin, Silove, Manicavasagar, \& Steel, 2004; Nixon, Resick, \& Nishith, 2004; Shalev et al., 1998), and increased health service utilization (Kramer, Booth, Han, \& Williams, 2003; Stapleton, Asmundson, Woods, Taylor, \& Stein, 2006) than individuals with either PTSD or MDD alone. Co-occurring PTSD and MDD has associated negative correlates and outcomes including increased risk for attempted suicide (Oquendo et al., 2005), a more chronic course of impairment and psychopathology (Breslau et al., 1991), and attenuated treatment response (Green et al., 2006). Overall, it is clear that MDD is jointly involved in the presentation of PTSD and that the co-occurrence is related to a more severe symptom presentation and disorder course.

What is not clear, however, is how to best understand theoretically the nature of the relationship between the constructs of PTSD and MDD. Such an understanding would lead to a clearer explanation of the high rates of co-occurrence and related greater symptom severity and impairment and, ultimately, could inform diagnostic classification and enhance treatment effectiveness. Symptom level analyses are a useful method in the development of a better theoretical understanding. In an attempt to reach a clearer understanding of the nature of this relationship, two key questions must be considered. The first question is whether or not PTSD and MDD are indeed separate constructs in response to trauma exposure. Following trauma, co-occurring PTSD and MDD may be best conceptualized as either two distinct constructs or a single general traumatic stress response. The second related question is whether the relationship between PTSD and MDD following trauma exposure is solely a function of shared symptoms. If PTSD and MDD are best represented as two distinct constructs, their co-occurrence may be the result of overlap between the constructs due to shared disorder features. Consideration of these two questions is an important step in moving towards a better understanding of the shared and unique trait and symptom dimensions driving the high rates of PTSD and MDD co-occurrence and related disorder severity.

With regard to the first question, to our knowledge, three studies have specifically examined whether PTSD and MDD represent two distinct responses to trauma or a general traumatic stress response (Blanchard et al., 1998; Grant, Beck, Marques, Palyo, \& Clapp, 2008; O'Donnell, Creamer, \& Pattison, 2004). Among samples of individuals who met diagnostic criteria for PTSD alone, MDD alone, or co-occurring PTSD and MDD, two studies using factor analyses evaluated and compared a single-factor model of posttraumatic stress symptoms (a unitary general traumatic stress response factor) to a two-factor (PTSD and MDD factors) or three-factor (PTSD, MDD, and generalized anxiety disorder factors) model of posttraumatic stress symptoms. The results of these studies suggested that PTSD and MDD might be best conceptualized as two independent, yet highly overlapping factors (Blanchard et al., 1998; Grant et al., 2008). In contrast, when exploring predictors of PTSD versus co-occurring PTSD and MDD, O'Donnell and colleagues (2004) found nearly identical sets of predictor variables for each, suggesting that PTSD and co-occurring PTSD and MDD may be essentially the same construct and that their separation may be an arbitrary distinction. Interestingly though, a different set of predictors differentiated MDD from both PTSD and co-occurring PTSD and MDD in the first few months post trauma. Such results argue that depression may exist as a distinct construct separate from a more general traumatic stress construct shortly after a traumatic event (O’Donnell et al., 2004). 
If PTSD and MDD are separate constructs in response to trauma exposure, their cooccurrence may be a function of shared symptoms. Prior research has approached this question in different ways. Examining PTSD and MDD diagnostic criteria, researchers have generally found that overlapping symptoms (e.g., insomnia, impaired concentration, anhedonia) are an insufficient explanation for the high rates of co-occurrence because the high rates of clinically significant co-occurring depression remain even after accounting for overlapping symptom items (e.g., Blanchard et al., 1998; Bleich, Koslowsky, Dolev, \& Lerer, 1997; Elhai, Grubaugh, Kashdan, \& Frueh, 2008; Taft, Resick, Watkins, \& Panuzio, 2009).

It is possible that the relationship between PTSD and MDD in response to trauma exposure is a function of shared dysphoria symptoms. Numerous studies have investigated the underlying structure of PTSD symptoms partly in response to the questioned validity of the three-cluster symptom structure specified by the DSM-IV (i.e., re-experiencing, avoidancenumbing, and hyperarousal; Simms, Watson, \& Doebbeling, 2002). Drawing on evidence that the anxiety and mood disorders share a general distress component, Simms and colleagues (2002) developed a PTSD symptoms structural model consisting of dysphoria, re-experiencing, avoidance, and hyperarousal factors. The dysphoria factor is composed of the inability to recall aspects of trauma, loss of interest, detachment, restricted affect, sense of foreshortened future, sleep disturbance, irritability, and difficulty concentrating. This four-factor model has since gained extensive empirical support (e.g., Baschnagel, O'Connor, Colder, \& Hawk, 2005; Elhai, Ford, Ruggiero, \& Frueh, 2009; Elklit \& Shevlin, 2007; Krause, Kaltman, Goodman, \& Dutton, 2007; Palmieri, Weathers, Difede, \& King, 2007). Further, studies examining associations among the four PTSD symptom factors and measures of depression have revealed that the depression measures are more strongly correlated with the dysphoria factor than with the other PTSD symptom factors (e.g., Elklit \& Shevlin, 2007; Palmieri et al., 2007; Simms et al., 2002). Thus, the dysphoria factor may partly explain the shared PTSD and MDD constructs.

From a broader perspective, a negative affect component (NA), present at varying levels in anxiety and mood disorders, has been shown to contribute to the overlap among these disorders (Brown, Chorpita, \& Barlow, 1998; Brown \& McNiff, 2009; Mineka, Watson, \& Clark, 1998). In addition, low positive affect (PA) has been shown to be a specific feature of depression that distinguishes MDD from the majority of the anxiety disorders (Brown et al., 1998; Mineka et al., 1998). In line with the dysphoria factor findings stated above, these specific affect components (NA and PA) may play differential roles in the co-occurring PTSD and MDD relationship. More specifically, negative affect may contribute to the overlap between the disorder constructs, while low positive affect may distinguish them. Indeed, a study examining mood and anxiety disorders in relation to higher order personality traits among a sample of Gulf War veterans found that negative temperament had the strongest associations with both MDD and PTSD and that positive temperament had a stronger negative correlation with MDD than with PTSD (Gamez, Watson, \& Doebbeling, 2007).

To better understand the relationship between co-occurring PTSD and MDD after trauma exposure, the present study examined whether data were more consistent with the possibility that PTSD and MDD are separate constructs and the extent to which the relationship between PTSD and MDD is a function of shared symptoms. We approached the first question by examining the effects of co-occurring MDD on disorder symptoms and impairment. More specifically, we examined whether the co-occurrence of PTSD and MDD would be associated with more severe PTSD and depressive symptoms and functional impairment than PTSD alone. We hypothesized that (1a) individuals with co-occurring PTSD and MDD would display more severe PTSD and depressive symptoms and functional 
impairment than individuals with PTSD alone. We also examined whether MDD is a separate, additional response to trauma exposure, rather than MDD symptoms simply being a marker of more severe posttraumatic stress symptoms. In this examination, we reasoned that if more severe depressive symptoms and functional impairment in co-occurring PTSD and MDD remained after holding PTSD constant, this would be consistent with MDD as a separate construct. We hypothesized that (1b) after controlling for PTSD severity, the cooccurrence of PTSD and MDD would still be associated with more severe depressive symptoms and functional impairment than PTSD alone. In testing this hypothesis, we controlled for interviewer-rated PTSD when comparing groups on interviewer-rated measures, and controlled for self-reported PTSD when comparing groups on self-report measures. This was done in order to control for shared error variance. Additionally, interview ratings may be more conservative in that they provide a tighter assessment of the construct of interest than self-report ratings. Finally, consistent results between analyses using interview and self-report ratings lend stronger support to study findings. Additionally, we further approached the first question by examining the roles of PTSD and depressive symptom severity and impairment in the prediction of co-occurring MDD. We hypothesized that (1c) depressive symptom severity would add to the prediction of co-occurring MDD beyond that of PTSD symptom severity and impairment.

We approached the second question by comparing the empirically-derived PTSD four symptom cluster solution (i.e., dysphoria, re-experiencing, avoidance, and hyperarousal; Simms et al., 2002) and levels of negative and positive affect between individuals with cooccurring PTSD and MDD and individuals with PTSD alone. We hypothesized that (2a) individuals with co-occurring PTSD and MDD would display elevated severity on only the dysphoria symptom cluster, as opposed to broader overlap across all symptom clusters, than those with PTSD alone and that (2b) individuals with co-occurring PTSD and MDD would display higher levels of negative affect and lower levels of positive affect than those with PTSD alone.

\section{Method}

\subsection{Participants}

The sample consisted of 173 treatment-seeking women $(78 \%, n=135)$ and men $(22 \%, n=$ 38 ) between the ages of 18 and 65 with a primary diagnosis of chronic PTSD. Of the 173 participants, 92 (53.2\%) were diagnosed with current co-occurring MDD. Participants were recruited via referrals and advertisements. Exclusion criteria included: a) current psychosis, unstable bipolar disorder, current substance dependence, or high suicide risk and b) in assault cases, an ongoing relationship with the perpetrator. On average, participants were $37.16(S D=11.09)$ years old. The average time since trauma was $11.58(S D=12.22)$ years. See Table 1 for sample characteristics.

\subsection{Measures}

Both interviewer-rated and self-report measures of PTSD, depression, and functional impairment were utilized because the inclusion of interviewer ratings decreases the bias that occurs with using self-report measures exclusively. Thus, the use of multiple measures allowed for a more comprehensive assessment of PTSD and co-occurring MDD diagnoses and symptom severity to be used in analyses to better understand the relationship between PTSD and MDD in response to trauma.

PTSD Symptom Scale - Interview (PSS-I)-The PSS-I (Foa, Riggs, Dancu, \& Rothbaum, 1993) is a semi-structured interview assessing PTSD diagnosis and severity and was used in this study to obtain both a diagnosis of primary chronic PTSD and to determine 
PTSD severity. The measure consists of 17 items corresponding to the DSM-IV PTSD symptoms. Items are rated on a 0 to 3 scale for combined frequency and severity in the past two weeks $(0=$ not at all, $3=5$ or more times per week/very much $)$. The PSS-I has good convergent validity with other PTSD interview measures (Foa \& Tolin, 2000). Inter-rater reliability for PTSD diagnosis $(\kappa=.91)$ and overall severity $(r=.97)$ ratings are excellent (Foa et al., 1993). In the present study, over $10 \%$ of cases were rerated for inter-rater reliability. Reliability based on consistency was high for PTSD severity scores $(I C C=.95)$ and PTSD diagnosis $(\kappa=1.00)$.

In the present study, the PSS-I symptom items were also arranged to form the empiricallyderived dysphoria, re-experiencing, avoidance, and hyperarousal symptom clusters as proposed by Simms and colleagues (2002). The dysphoria symptom cluster consists of inability to recall aspects of trauma, loss of interest, detachment, restricted affect, sense of foreshortened future, sleep disturbance, irritability, and difficulty concentrating; the reexperiencing symptom cluster consists of intrusive thoughts of trauma, recurrent dreams of trauma, flashbacks, emotional reactivity to trauma cues, and physiological reactivity to trauma cues; the avoidance symptom cluster consists of avoiding thoughts of trauma and avoiding reminders of trauma; the hyperarousal symptom cluster consists of hypervigilance and exaggerated startle response.

Hamilton Rating Scale for Depression - 24 Items (HRSD $\left.{ }_{24}\right)$-The $\mathrm{HRSD}_{24}$ (Hamilton, 1960) is an interviewer-rated depressive symptom scale consisting of 24 items measuring the severity of cognitive, behavioral, and somatic symptoms of depression in the past week. Items are scored on either a 0 to 2 or 0 to 4 spectrum, with higher scores indicating greater severity. The HRSD has excellent inter-rater reliability $(r=.90)$ and good internal consistency ( $\alpha=.76$; Rehm \& O'Hara, 1985), and adequate convergent validity with a wide range of depression measures (Bagby, Ryder, Schuller, \& Marshall, 2004). The total $\mathrm{HRSD}_{24}$ score was included in this study as a measure of overall depression severity. In the present study, reliability for $\mathrm{HRSD}_{24}$ overall severity scores was good $(I C C=.89)$ based on the $10 \%$ of cases that were rerated for reliability.

\section{Structured Clinical Interview for DSM-IV Axis I (SCID-I)-The SCID-I (First,} Spitzer, Gibbon, \& Williams, 2001) is a diagnostic interview used to acquire information about DSM-IV Axis I disorder criteria. The SCID-I has acceptable joint interview inter-rater reliability with kappas between .57 and 1.0 (Zanarini et al., 2000). In this study, it was used to obtain a diagnosis of current MDD. In the present study, over $10 \%$ of the cases were rerated for diagnostic reliability. Overall, reliability was high with good diagnostic agreement for current MDD $(\kappa=.73)$.

Social Adjustment Scale (SAS)—The SAS (Weissman \& Paykel, 1974) is a semistructured interview used to assess an individual's functioning in eight specific areas (e.g., work, family unit) over the past two weeks. Individual and global items are measured on a scale ranging from 1 to 7 , with higher scores indicating more severe maladjustment. In this study, the overall adjustment score of global functioning was used to assess impairment in functioning. The SAS has good inter-rater reliability $(r=.80$; Weissman, Paykel, Siegel, \& Klerman, 1971). In this study, reliability for global functioning scores was adequate (ICC $=$. 62 ) based on the $10 \%$ of cases that were rerated for reliability.

PTSD Symptom Scale - Self-Report (PSS-SR)—The PSS-SR (Foa et al., 1993) is a 17-item version of the Posttraumatic Stress Diagnostic Scale (PDS; Foa, Cashman, Jaycox, $\&$ Perry, 1997), assessing the severity of DSM-IV PTSD symptoms. Each symptom is rated on a 4-point scale from 0 (not at all) to 3 (very much), with higher scores indicating more severe PTSD symptoms. This measure has high internal consistency $(\alpha=.91)$, excellent 
inter-rater reliability for PTSD diagnosis $(\kappa=.91)$ and overall severity $(r=.97)$, and good test-retest reliability for the total score $(r=.83$; Foa et al., 1993).

Beck Depression Inventory (BDI)—The BDI (Beck, Ward, Mendelsohn, Mock, \& Erbaugh, 1961) is a 21-item self-report inventory measuring depression severity over the past week. Each item consists of statements scored 0 to 3, with increasing scores indicating greater severity of depression. The BDI has a split-half reliability of .93 (Beck et al., 1961) and correlates strongly with clinical ratings of depression $(r=.55$ to .96 ; Beck, Steer, \& Carbin, 1988).

\section{Positive and Negative Affect Schedule - Trait Version (PANAS)—The PANAS}

(Watson, Clark, \& Tellegen, 1988) consists of two 10-item scales measuring the two primary dimensions of mood, positive and negative affect. Participants rate on a 5-point scale $(1=$ very slightly or not at all, $5=$ extremely $)$ to what extent they experience each mood state in general (i.e., trait). The PANAS scales are stable over a 2-month time period ( $\alpha=.47$ to .68 for PA and .39 to .71 for NA), highly internally consistent ( $\alpha=.86$ to .90 for PA and .84 to .87 for NA), and largely uncorrelated ( $r=-.12$ to -.23 ; Watson et al., 1988). The PANAS has high convergent validity with lengthier measures of $\operatorname{mood}(r=-.19$ to -.36 for PA and .51 to .74 for NA; Watson et al., 1988).

Sheehan Disability Scale (SDS)—The SDS (Sheehan, 1983) is a 3-item self-report scale of impairment. The items are scored on a 0 to 10 scale with increasing scores indicating greater impairment, and address the impact of symptomatology on three areas of functioning: work, social, and family. The total impairment score is the unweighted sum of the 3 items. In this study, the total impairment score was used to assess impairment in functioning. The instrument has adequate reliability ( $\alpha=.56$ to .86$)$ and construct validity as evaluated by examining the sensitivity of the instrument to change (Cohen's $d=.79$ to 1.46 ; Leon, Shear, Portera, \& Klerman, 1992).

\section{Results}

\subsection{Preliminary Analyses}

Comparisons between the co-occurring PTSD and MDD (PTSD+MDD) group and the PTSD group on demographics were performed using the chi-square test for the categorical variables (i.e., sex, trauma type, education level, income, and ethnicity) and the independentsamples $t$-test for the continuous variables age and time since trauma. There was a significant difference in age between the groups. Individuals with PTSD+MDD $(M=39.41$, $S D=11.09)$ were older than those with PTSD alone $(M=34.59, S D=10.58), t(171)=$ $-2.91, p<.01, d=.44$. No other significant differences in demographics emerged. The demographic variable age was evaluated as a potential covariate for the subsequent analyses of covariance and is reported in the text if findings were altered by its inclusion.

\subsection{PTSD and Depressive Symptom Severity and Functional Impairment between PTSD +MDD and PTSD Alone}

Independent-samples $t$-tests were conducted to test the hypothesis that (1a) individuals with PTSD+MDD would display more severe PTSD and depressive symptoms and functional impairment than individuals with PTSD alone. As seen in Table 2, across all symptom severity and functioning measures, individuals with PTSD+MDD were more severe than those with PTSD alone. 


\subsection{Controlling of PTSD Severity: Depressive Symptom Severity and Functional Impairment between PTSD+MDD and PTSD Alone}

In order to test the hypothesis that (1b) after controlling for PTSD severity, PTSD+MDD would continue to be associated with more severe depressive symptoms and functional impairment than PTSD alone, one-way between-group analyses of covariance (ANCOVAs) were conducted to compare depressive symptom severity and functional impairment between PTSD+MDD and PTSD alone after adjusting for PTSD symptom severity. After adjusting for interviewer-rated PTSD symptom severity scores (PSS-I), individuals with PTSD+MDD $\left(M_{\text {adjusted }}=27.11, S E=.802\right)$ still had higher interviewer-rated depressive symptom severity scores on the $\mathrm{HRSD}_{24}$ than PTSD $\left(M_{\text {adjusted }}=20.05, S E=.860\right), F(1$, $170)=32.90, p<.001, d=.88$. Similarly, after adjusting for self-reported PTSD symptom severity scores (PSS-SR), individuals with PTSD+MDD ( $M_{\text {adjusted }}=26.35, S E=.804$ ) had significantly higher self-reported depressive symptom severity scores on the BDI than those with PTSD alone $\left(M_{\text {adjusted }}=23.22, S E=.851\right), F(1,168)=6.64, p<.05, d=.39$.

In contrast to depression severity, for interviewer-rated global functioning (SAS), after controlling for PTSD severity (PSS-I), there was no difference between those with PTSD $+\mathrm{MDD}\left(M_{\text {adjusted }}=3.84, S E=.104\right)$ and those with PTSD alone $\left(M_{\text {adjusted }}=3.78, S E=\right.$. $112), F(1,170)=.15$, ns. Similarly, for self-reported total impairment (SDS), after controlling for both PTSD severity (PSS-SR) and age, there was no difference between those with PTSD+MDD $\left(M_{\text {adjusted }}=19.22, S E=.612\right)$ and those with PTSD alone $\left(M_{\text {adjusted }}\right.$ $=18.00, S E=.656), F(1,169)=1.68, n s$.

For further exploration, hierarchical multiple regression was used to assess how much of the variance in functional impairment was explained by PTSD symptom severity, depressive symptom severity, and the overlap between the two. For both interviewer-rated and selfreport measures, two separate hierarchical multiple regression analyses were conducted. The first examined the percentage of the variance in impaired functioning explained by depressive symptom severity after controlling for the effect of PTSD symptom severity. The second examined the percentage of the variance in impaired functioning explained by PTSD symptom severity after controlling for the effect of depressive symptom severity. In order to examine the percentage of the variance in impaired functioning that was shared between PTSD symptom severity and depressive symptom severity, the PTSD symptom severity unique variance and depressive symptom severity unique variance were summed and that total was subtracted from the total variance explained by the model as a whole. The detailed results of the hierarchical multiple regression analyses for interviewer-rated and self-report measures can be seen in Table 3. In examining overlap between PTSD and depressive symptom severity, $17.3 \%$ of the variance in interviewer-rated functional impairment (SAS) was shared between PTSD symptom severity (PSS-I) and depressive symptom severity $\left(\mathrm{HRSD}_{24}\right)$. The total variance shared between PTSD symptom severity (PSS-SR) and depressive symptom severity (BDI) in self-reported functional impairment (SDS) was 26.7\%. Overall, the overlap between PTSD and depressive symptom severity appears to explain a larger percentage of the variance in functional impairment than either PTSD symptom severity or depressive symptom severity.

\subsection{PTSD and Depressive Symptom Severity and Functional Impairment in the Prediction of Co-occurring MDD}

Sequential logistic regressions were conducted in order to test the hypothesis that (1c) depressive symptom severity would add to the prediction of co-occurring MDD beyond that of PTSD symptom severity, functional impairment, and age. The two measures of PTSD symptom severity, the PSS-I and PSS-SR, were centered for the analyses. Interviewer-rated PTSD symptom severity (PSS-I), functional impairment (SAS), and age were entered in 
block 1 and interviewer-rated depressive symptom severity $\left(\mathrm{HRSD}_{24}\right)$ was entered in block 2 . The first block resulted in a significant model $\chi^{2}(3, N=173)=41.49, p<.001$. The $\mathrm{HRSD}_{24}$ significantly added to prediction of co-occurring MDD above that afforded by PSS-I, SAS, and age, $\chi^{2}(1, N=173)=28.52, p<.001$. With all of the predictors included (i.e., PSS-I, SAS, age, and $\left.\mathrm{HRSD}_{24}\right)$, according to the Wald criterion, only the $\operatorname{HRSD}_{24}(z=$ $22.90, p<.001, \mathrm{OR}=1.14)$ and age $(z=4.77, p<.05$, OR $=1.04)$ reliably predicted cooccurring MDD. In the second regression, self-reported PTSD symptom severity (PSS-SR), functional impairment (SDS), and age were entered in block 1 and self-reported depressive symptom severity (BDI) was entered in block 2 . The first block resulted in a significant model $\chi^{2}(3, N=171)=34.30, p<.001$. The BDI significantly added to prediction of cooccurring MDD above that afforded by PSS-SR, SDS, and age, $\chi^{2}(1, N=171)=4.43, p<$. 05 . With all of the predictors included (i.e., PSS-SR, SDS, age, and BDI), the PSS-SR $(z=$ $4.58, p<.05, \mathrm{OR}=1.06)$, age $(z=4.20, p<.05, \mathrm{OR}=1.03)$, and the $\mathrm{BDI}(z=4.34, p<.05$, $\mathrm{OR}=1.06)$ reliably predicted co-occurring MDD. Overall, these results show that depressive symptoms play a unique role in the presence of co-occurring MDD.

\subsection{PTSD Symptom Clusters and Affective Components Comparisons between PTSD +MDD and PTSD Alone}

To examine the hypothesis that (2a) individuals with PTSD+MDD would display elevated severity on the dysphoria PTSD symptom cluster only as compared to those with PTSD alone, independent-samples $t$-tests were conducted to compare the severity of the four empirically-derived symptom clusters (i.e., dysphoria, re-experiencing, avoidance, and hyperarousal; Simms et al., 2002) for PTSD+MDD and PTSD alone. As expected and as can be seen in Table 4, individuals with PTSD+MDD had more severe symptoms on the dysphoria symptom cluster than those with PTSD alone $(d=1.12)$. An unexpected finding was that this was also the case for the re-experiencing symptom cluster, though a much smaller effect $(d=.41)$. Consistent with fear-based symptoms being characteristic of PTSD, no significant differences between the two groups were seen on the avoidance symptom cluster or hyperarousal symptom cluster severity.

Finally, consistent with our hypothesis ( $2 \mathrm{~b}$ ) regarding levels of affect, as seen in Table 4, levels of NA were significantly higher and levels of PA were significantly lower for individuals with PTSD+MDD compared to those with PTSD alone.

\section{Discussion}

Across measures, the nature of the relationship between PTSD and MDD in response to trauma exposure was more consistent with a model of two separate, yet overlapping constructs than a single general traumatic stress response. The investigations of PTSD and MDD symptom severity and functional impairment suggest that PTSD and MDD cooccurrence may be more than simply a marker of posttraumatic stress severity. The comparisons between individuals with co-occurring PTSD and MDD and individuals with PTSD alone on levels of trauma-related symptom clusters and affect suggest that shared symptoms may be a partial explanation for the PTSD and MDD co-occurrence. Taken together, PTSD and MDD following trauma may be two separate constructs contributing to greater symptom severity with their co-occurrence being a function of shared symptom and affective components.

Individuals with co-occurring PTSD and MDD reported more severe PTSD, depressive symptoms, and functional impairment than individuals with PTSD alone. The co-occurrence of PTSD and MDD was associated with more depressive symptom severity even after controlling for PTSD severity. Thus, MDD appears to uniquely impact posttraumatic depressive symptom severity among individuals diagnosed with co-occurring PTSD and 
MDD. Further, depressive symptom severity added to the prediction of co-occurring MDD beyond that of PTSD symptom severity, functional impairment, and age, suggesting that depressive symptoms play an important role in the presence of co-occurring MDD. In line with previous studies (Blanchard et al., 1998; Grant et al., 2008), these findings lend support to the idea that PTSD and MDD may indeed be separate constructs.

Interestingly, when holding PTSD severity constant, the more severe functional impairment, as measured by social functioning and disability, found among individuals with co-occurring PTSD and MDD was no longer present, possibly suggesting that PTSD symptom severity may be partly responsible for the worse functional impairment when PTSD and MDD cooccur. Indeed, the amount of variance in functional impairment explained by PTSD symptom severity and depressive symptom severity was similar and the shared variance between the two was even higher. This result is in line with previous work finding that PTSD contributed substantially to impaired functioning and diminished quality of life even after adjusting for co-occurring depression (Zatzick et al., 1997). Additionally, the lack of difference in functioning between individuals with co-occurring PTSD and MDD and individuals with PTSD alone after controlling for PTSD symptom severity further supports the idea that MDD as a separate construct uniquely impacts posttraumatic depressive symptom severity because the more severe depressive symptoms are not solely a function of general functional impairment.

Notably, the relationship between PTSD and MDD appears to be partly a function of shared dysphoria or general distress symptoms. Individuals with co-occurring PTSD and MDD displayed elevated severity on the dysphoria symptom cluster, but not on the more fearbased trauma-related avoidance and hyperarousal symptom clusters, suggesting that the dysphoria symptoms of the PTSD construct may be shared with specific symptoms of the MDD construct (Palmieri et al., 2007; Simms et al., 2002). No significant difference between individuals with co-occurring PTSD and MDD and individuals with PTSD alone on avoidance and hyperarousal symptom cluster severity suggests that the PTSD construct contains anxiety disorder specific fear-based symptom components that may help distinguish the PTSD and MDD constructs. However, contrary to our predictions, individuals with co-occurring PTSD and MDD also displayed elevated severity on the reexperiencing symptom cluster, though at a more modest level than the dysphoria symptom cluster. Elevated severity on the re-experiencing symptom cluster among individuals with co-occurring PTSD and MDD may be due to the similarity between traumatic intrusions in PTSD and trauma-related rumination found in MDD (Ehring, Frank, \& Ehlers, 2008). With current PTSD criteria not providing good guidance on traumatic intrusions and rumination differentiation, such as distinguishing between the intrusive cognitions of remembering the trauma (i.e., re-experiencing) and repetitively thinking about the trauma (i.e., rumination; Ehlers \& Clark, 2000), the elevated re-experiencing symptoms may have resulted from rumination.

With co-occurring PTSD and MDD characterized by higher levels of negative affect and lower levels of positive affect, a shared negative affect component may also contribute to PTSD and MDD co-occurrence, while lack of positive affect may further support the idea of MDD and PTSD as separate constructs following trauma exposure. Indeed, these findings are consistent with dimensional models (Brown et al., 1998; Clark \&Watson, 1991; Gamez et al., 2007; Mineka et al., 1998; Watson, 2005; 2009) that present the notion of a negative affect component as a shared feature among the anxiety and mood disorders and responsible for the high rates of co-occurrence, and a low positive affect component as a specific, distinguishing feature of depression. Further, although not examined in the current study because of an absence of specific measures, the models also include anxiety disorder specific components, such as the empirically supported physiological hyperarousal 
component (Joiner et al., 1999) that may be a specific and distinguishing feature of PTSD and panic disorder (Brown \& McNiff, 2009).

Given that this study was focused on individuals with a primary diagnosis of chronic PTSD, individuals with MDD without trauma exposure or MDD without PTSD, but with trauma exposure were not included. Therefore, we were unable to parse out the role of trauma exposure or MDD alone in PTSD and depressive symptom severity and levels of affect. The possibility of a lack of severity difference between MDD and co-occurring PTSD and MDD is unlikely, however, as previous studies (e.g., Shalev et al., 1998) have shown that individuals with co-occurring PTSD and MDD tend to report more symptoms and greater impairment than those with MDD alone. We also did not consider the other characteristics beyond presence and severity that may be important in understanding the underlying relationship between PTSD and MDD. It is possible that the time of onset for MDD or recurrent nature of MDD may affect the impact of specific depressive symptoms on overall co-occurring PTSD and MDD disorder severity. For example, the presence of depression before a traumatic event has been associated with more depression and PTSD symptoms across time (Shalev et al., 1998). However, studies (e.g., Blanchard et al., 1998) have shown that co-occurring PTSD and MDD has more severe symptoms even when MDD occurs at different times of onset. Rather, the inability to disengage from a negative mood state and the tendency to easily move back into that state is a more likely marker of depression (Holtzheimer \& Mayberg, 2011).

A clearer understanding of the nature of the relationship between PTSD and MDD in response to trauma exposure has important clinical implications. The more severe dysphoria symptom cluster, elevated levels of negative affect, and lack of positive affect among individuals with co-occurring PTSD and MDD as compared to individuals with PTSD alone have implications for the PTSD diagnostic criteria in the DSM-V. More specifically, perhaps a dysphoria symptom cluster should be recognized in PTSD and subsequently given less diagnostic weight in relation to the fear-based symptom clusters (i.e., avoidance and hyperarousal) in order to promote differentiation from disorders with overlapping distress symptoms, such as MDD. As Watson (2009) noted, "it is not clear that it is necessary - or even desirable - to build such a strong distress component into the diagnosis of PTSD” (p. 242). Thus, in order to capture PTSD and MDD as two distinct disorders, perhaps less emphasis should be placed on the overlapping disorder features, that is, dysphoria symptoms, in comparison to the distinguishing disorder features, that is, fear-based symptoms in PTSD and low positive affect in depression.

Future theoretical work is needed to help clarify the nature of the relationship between PTSD and MDD following trauma exposure. More specifically, examination of the latent structure of PTSD and MDD within a structural model of co-occurrence will aid in determining whether PTSD and MDD are indeed distinct, yet overlapping constructs in response to trauma.

\section{Acknowledgments}

Role of the funding source

Preparation of this manuscript was supported by grants to Drs. Zoellner and Feeny from the National Institute of Mental Health (R01 MH066347, R01 MH066348) and the DCRU National Institute of Health (M01 RR00080). Neither the National Institute of Mental Health nor the DCRU National Institute of Health had involvement in this study's design; in the collection, analysis, and interpretation of data; in the writing of this report; or in the decision to submit this paper for publication. 


\section{References}

Bagby RM, Ryder AG, Schuller DR, Marshall MB. The Hamilton Depression Rating Scale: Has the gold standard become a lead weight? American Journal of Psychiatry. 2004; 161:2163-2177. [PubMed: 15569884]

Baschnagel JS, O'Connor RM, Colder CR, Hawk LW Jr. Factor structure of posttraumatic stress among western New York undergraduates following the September $11^{\text {th }}$ terrorist attack on the World Trade Center. Journal of Traumatic Stress. 2005; 18:677-684. [PubMed: 16382430]

Beck AT, Steer RA, Carbin MG. Psychometric properties of the Beck Depression Inventory: Twentyfive years of evaluation. Clinical Psychology Review. 1988; 8:77-100.

Beck AT, Ward CH, Mendelsohn M, Mock J, Erbaugh J. An inventory for measuring depression. Archives of General Psychiatry. 1961; 4:561-571. [PubMed: 13688369]

Blanchard EB, Buckley TC, Hickling EJ, Taylor AE. Posttraumatic stress disorder and comorbid major depression: Is the correlation an illusion? Journal of Anxiety Disorders. 1998; 12:21-37. [PubMed: 9549607]

Bleich A, Koslowsky M, Dolev A, Lerer B. Post-traumatic stress disorder and depression: An analysis of comorbidity. British Journal of Psychiatry. 1997; 170:479-482. [PubMed: 9307701]

Breslau N, Davis GC, Andreski P, Peterson E. Traumatic events and posttraumatic stress disorder in an urban population of young adults. Archives of General Psychiatry. 1991; 48:216-222. [PubMed: 1996917]

Breslau N, Davis GC, Peterson EL, Schultz L. Psychiatric sequelae of posttraumatic stress disorder in women. Archives of General Psychiatry. 1997; 54:81-87. [PubMed: 9006404]

Brown TA, Campbell LA, Lehman CL, Grisham JR, Mancill RB. Current and lifetime comorbidity of the DSM-IV anxiety and mood disorders in a large clinical sample. Journal of Abnormal Psychology. 2001; 110:585-599. [PubMed: 11727948]

Brown TA, Chorpita BF, Barlow DH. Structural relationships among dimensions of the DSM-IV anxiety and mood disorders and dimensions of negative affect, positive affect, and autonomic arousal. Journal of Abnormal Psychology. 1998; 107:179-192. [PubMed: 9604548]

Brown TA, McNiff J. Specificity of autonomic arousal to DSM-IV panic disorder and posttraumatic stress disorder. Behaviour Research and Therapy. 2009; 47:487-493. [PubMed: 19321162]

Clark LA, Watson D. Tripartite model of anxiety and depression: Psychometric evidence and taxonomic implications. Journal of Abnormal Psychology. 1991; 100:316-336. [PubMed: 1918611]

Davidson JR, Hughes D, Blazer DG, George LK. Post-traumatic stress disorder in the community: An epidemiological study. Psychological Medicine. 1991; 21:713-721. [PubMed: 1946860]

Ehlers A, Clark DM. A cognitive model of posttraumatic stress disorder. Behaviour Research and Therapy. 2000; 38:319-345. [PubMed: 10761279]

Ehring T, Frank S, Ehlers A. The role of rumination and reduced concreteness in the maintenance of posttraumatic stress disorder and depression following trauma. Cognitive Therapy and Research. 2008; 32:488-506. [PubMed: 20694036]

Elhai JD, Ford JD, Ruggiero KJ, Frueh BC. Diagnostic alterations for post-traumatic stress disorder: Examining data from the National Comorbidity Survey Replication and National Survey of Adolescents. Psychological Medicine. 2009; 39:1957-1966. [PubMed: 19379536]

Elhai JD, Grubaugh AL, Kashdan TB, Frueh BC. Empirical examination of a proposed refinement to DSM-IV posttraumatic stress disorder symptom criteria using the National Comorbidity Survey Replication data. Journal of Clinical Psychiatry. 2008; 69:597-602. [PubMed: 18294026]

Elklit A, Shevlin M. The structure of PTSD symptoms: A test of alternative models using confirmatory factor analysis. British Journal of Clinical Psychology. 2007; 46:299-313. [PubMed: 17535523]

First, MB.; Spitzer, RL.; Gibbon, M.; Williams, JBW. Structured Clinical Interview for DSM-IV-TR Axis I Disorders, Research Version, Patient Edition With Psychotic Screen (SCID-I/P w/PSY SCREEN). New York: Biometrics Research, New York State Psychiatric Institute; 2001.

Foa EB, Cashman L, Jaycox L, Perry K. The validation of a self-report measure of posttraumatic stress disorder: The Posttraumatic Diagnostic Scale. Psychological Assessment. 1997; 9:445-451. 
Foa EB, Riggs DS, Dancu CV, Rothbaum BO. Reliability and validity of a brief instrument for assessing posttraumatic stress disorder. Journal of Traumatic Stress. 1993; 6:459-473.

Foa EB, Tolin DF. Comparison of the PTSD Symptom Scale - Interview Version and the ClinicianAdministered PTSD Scale. Journal of Traumatic Stress. 2000; 13:181-191. [PubMed: 10838669]

Gamez W, Watson D, Doebbeling BN. Abnormal personality and the mood and anxiety disorders: Implications for structural models of anxiety and depression. Journal of Anxiety Disorders. 2007; 21:526-539. [PubMed: 16978832]

Grant DM, Beck JG, Marques L, Palyo SA, Clapp JD. The structure of distress following trauma: Posttraumatic stress disorder, major depressive disorder, and generalized anxiety disorder. Journal of Abnormal Psychology. 2008; 117:662-672. [PubMed: 18729617]

Green BL, Krupnick JL, Chung J, Siddique J, Krause ED, Revicki D, Miranda J. Impact of PTSD comorbidity on one-year outcomes in a depression trial. Journal of Clinical Psychology. 2006; 62:815-835. [PubMed: 16703602]

Hamilton M. A rating scale for depression. Journal of Neurology Neurosurgery and Psychiatry. 1960; 23:56-62.

Hankin CS, Spiro A, Miller DR, Kazis L. Mental disorders and mental health treatment among U.S. Department of Veterans Affairs outpatients: The Veterans Health Study. American Journal of Psychiatry. 1999; 156:1924-1930. [PubMed: 10588406]

Helzer JE, Robins LN, McEvoy L. Post-traumatic stress disorder in the general population. New England Journal of Medicine. 1987; 317:1630-1634. [PubMed: 3683502]

Holtzheimer PE, Mayberg HS. Stuck in a rut: Rethinking depression and its treatment. Trends in Neurosciences. 2011; 34:1-9. [PubMed: 21067824]

Joiner TE Jr, Steer RA, Beck AT, Schmidt NB, Rudd MD, Catanzaro SJ. Physiological hyperarousal: Construct validity of a central aspect of the tripartite model of depression and anxiety. Journal of Abnormal Psychology. 1999; 108:290-298. [PubMed: 10369039]

Kessler RC, Berglund P, Demler O, Jin R, Walters EE. Lifetime prevalence and age-of-onset distributions of DSM-IV disorders in the National Comorbidity Survey Replication. Archives of General Psychiatry. 2005; 62:593-602. [PubMed: 15939837]

Kessler RC, Chiu WT, Demler O, Walters EE. Prevalence, severity, and comorbidity of 12-month DSM-IV disorders in the National Comorbidity Survey Replication. Archives of General Psychiatry. 2005; 62:617-627. [PubMed: 15939839]

Kessler RC, Sonnega A, Bromet E, Hughes M, Nelson CB. Posttraumatic stress disorder in the National Comorbidity Survey. Archives of General Psychiatry. 1995; 52:1048-1060. [PubMed: 7492257]

Kramer TL, Booth BM, Han X, Williams DK. Service utilization and outcomes in medically ill veterans with posttraumatic stress and depressive disorders. Journal of Traumatic Stress. 2003; 16:211-219. [PubMed: 12816332]

Krause ED, Kaltman S, Goodman LA, Dutton MA. Longitudinal factor structure of posttraumatic stress symptoms related to intimate partner violence. Psychological Assessment. 2007; 19:165175. [PubMed: 17563198]

Leon AC, Shear MK, Portera L, Klerman GL. Assessing impairment in patients with panic disorder: The Sheehan Disability Scale. Social Psychiatry and Psychiatric Epidemiology. 1992; 27:78-82. [PubMed: 1594977]

Mineka S, Watson D, Clark LA. Comorbidity of anxiety and unipolar mood disorders. Annual Review of Psychology. 1998; 49:377-412.

Momartin S, Silove D, Manicavasagar V, Steel Z. Comorbidity of PTSD and depression: Associations with trauma exposure, symptom severity and functional impairment in Bosnian refugees resettled in Australia. Journal of Affective Disorders. 2004; 80:231-238. [PubMed: 15207936]

Nixon RDV, Resick PA, Nishith P. An exploration of comorbid depression among female victims of intimate partner violence with posttraumatic stress disorder. Journal of Affective Disorders. 2004; 82:315-320. [PubMed: 15488264]

O'Donnell ML, Creamer M, Pattison P. Posttraumatic stress disorder and depression following trauma: Understanding comorbidity. American Journal of Psychiatry. 2004; 161:1390-1396. [PubMed: 15285964] 
Oquendo M, Brent DA, Birmaher B, Greenhill L, Kolko D, Stanley B, Mann JJ. Posttraumatic stress disorder comorbid with major depression: Factors mediating the association with suicidal behavior. American Journal of Psychiatry. 2005; 162:560-566. [PubMed: 15741474]

Palmieri PA, Weathers FW, Difede J, King DW. Confirmatory factor analysis of the PTSD Checklist and the Clinician-Administered PTSD Scale in disaster workers exposed to the World Trade Center Ground Zero. Journal of Abnormal Psychology. 2007; 116:329-341. [PubMed: 17516765]

Rehm LP, O'Hara MW. Item characteristics of the Hamilton Rating Scale for Depression. Journal of Psychiatric Research. 1985; 19:31-41. [PubMed: 3989736]

Shalev AY, Freedman S, Peri T, Brandes D, Sahar T, Orr SP, Pitman RK. Prospective study of posttraumatic stress disorder and depression following trauma. American Journal of Psychiatry. 1998; 155:630-637. [PubMed: 9585714]

Sheehan, DV. The anxiety disease. New York: Scribner; 1983.

Shore JH, Vollmer WM, Tatum EL. Community patterns of posttraumatic stress disorders. Journal of Nervous and Mental Disease. 1989; 177:681-685. [PubMed: 2809579]

Simms LJ, Watson D, Doebbeling BN. Confirmatory factor analyses of posttraumatic stress symptoms in deployed and nondeployed veterans of the Gulf War. Journal of Abnormal Psychology. 2002; 111:637-647. [PubMed: 12428777]

Stapleton JA, Asmundson GJG, Woods M, Taylor S, Stein MB. Health care utilization by United Nations peacekeeping veterans with co-occurring, self-reported, post-traumatic stress disorder and depression symptoms versus those without. Military Medicine. 2006; 161:562-566. [PubMed: 16808142]

Taft CT, Resick PA, Watkins LE, Panuzio J. An investigation of posttraumatic stress disorder and depression symptomatology among female victims of interpersonal trauma. Journal of Family Violence. 2009; 24:407-415. [PubMed: 21052546]

Watson D. Rethinking the mood and anxiety disorders: A quantitative hierarchical model for DSM-V. Journal of Abnormal Psychology. 2005; 114:522-536. [PubMed: 16351375]

Watson D. Differentiating the mood and anxiety disorders: A quadripartite model. Annual Review of Clinical Psychology. 2009; 5:221-247.

Watson D, Clark LA, Tellegen A. Development and validation of brief measures of positive and negative affect: The PANAS scales. Journal of Personality and Social Psychology. 1988; 54:10631070. [PubMed: 3397865]

Weissman, MM.; Paykel, ES. The depressed woman: A study of social relations. Chicago: University of Chicago Press; 1974.

Weissman MM, Paykel ES, Siegel R, Klerman GL. The social role performance of depressed women: Comparisons with a normal group. American Journal of Orthopsychiatry. 1971; 41:391-405.

Zanarini MC, Skodol AE, Bender D, Dolan R, Sanislow C, Schaefer E, Gunderson JG. The Collaborative Longitudinal Personality Disorders Study: Reliability of Axis I and II diagnoses. Journal of Personality Disorders. 2000; 14:291-299. [PubMed: 11213787]

Zatzick DF, Marmar CR, Weiss DS, Browner WS, Metzler TJ, Golding JM, Wells KB. Posttraumatic stress disorder and functioning and quality of life outcomes in a nationally representative sample of male Vietnam veterans. American Journal of Psychiatry. 1997; 154:1690-1695. [PubMed: 9396947] 


\section{Highlights}

- The presence of MDD is not solely a function of PTSD severity

- PTSD and MDD share dysphoria symptoms and negative affect

- PTSD and MDD may be two distinct constructs with overlapping distress components 


\section{Table 1}

Sample Characteristics

\begin{tabular}{lc}
\hline Demographic Variable & Percentage \\
\hline Trauma Type & 32.9 \\
Adult Sexual Assault & 20.2 \\
Adult Non-Sexual Assault & 17.3 \\
Childhood Sexual Assault & 13.9 \\
Accident (MVA, natural disaster) & 6.9 \\
Childhood Non-Sexual Assault & 6.4 \\
Death/Violence to Loved One & 2.3 \\
Combat/War & \\
Education Level & 67.6 \\
Not College Educated & \\
Income & 47.4 \\
Less than \$20,000 per year & 68.2 \\
Ethnicity & 12.7 \\
Caucasian & \\
African American & \\
Other & \\
\hline
\end{tabular}




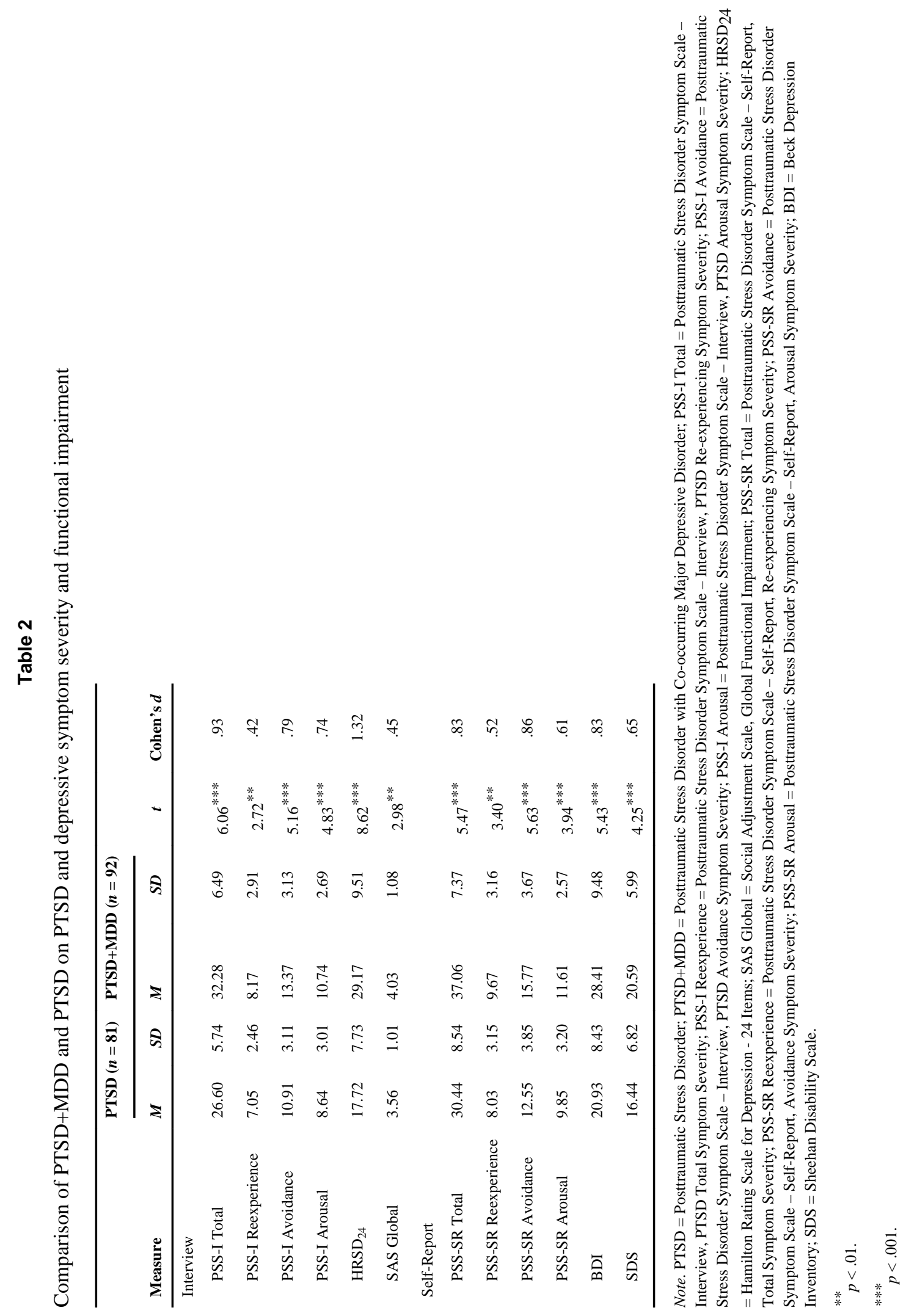




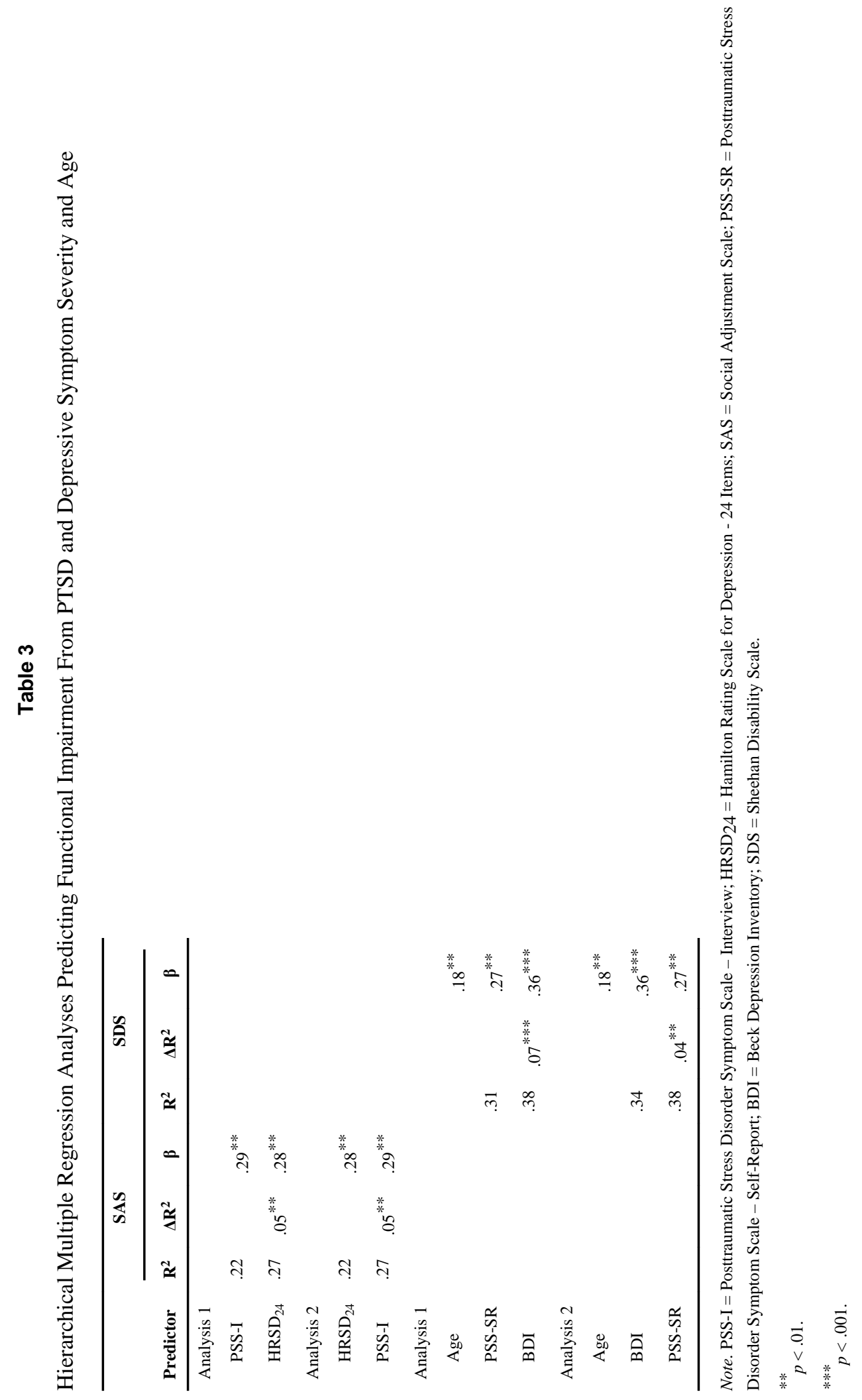




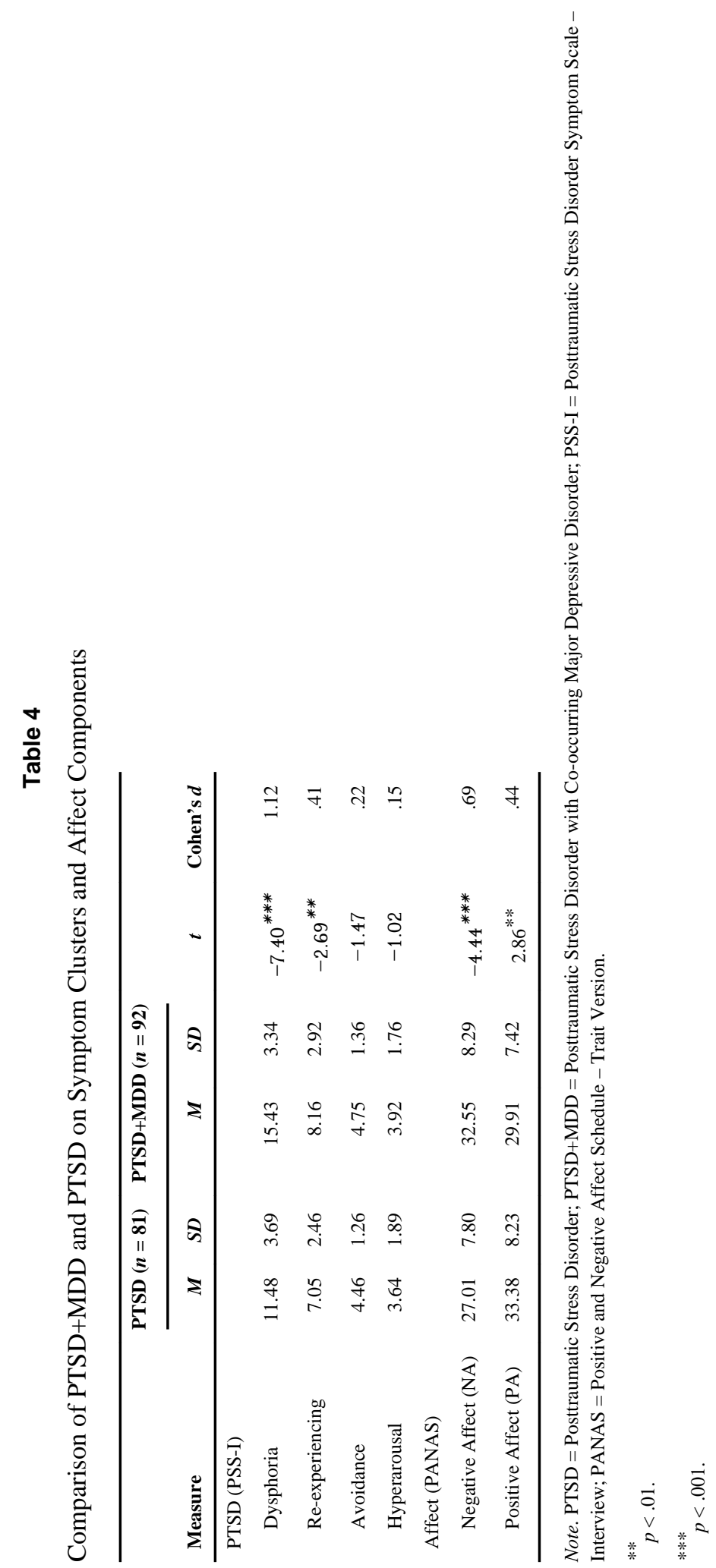

